\title{
ölkrise, Energiekrise oder Krise des Kapitalismus?
}

Vorbemerkung zu den nachfolgenden Aufsätzen von M. Massarrat und A. Tahmassebi

Die PROKLA druckt nachfolgend zwei Aufsätze zu den Problemen der sog. ,ölkri$\mathrm{se}^{\mathrm{a}} \mathrm{ab}$. Im Beitrag von M. Massarrat wird der Versuch unternommen, auf der Grundlage der Marxschen Wert theorie die gegenwärtige Auseinandersetzung um Ölpreis und Verteilung des Profits zwischen ölproduzierenden Ländern einerseits, multinationalen Ölkonzernen und hochentwickelten kapitalistischen Staaten andererseits politisch einzuschätzen. Im Aufsatz von A. Tahmassebi hingegen wird eher versucht, durch eine Untersuchung der politischen Entwicklung und Verhältnisse in den arabischen ölproduzierenden Staaten und durch eine Einschätzung der politischen Machtverhältnisse die Chancen für eine Umverteilung der bei der Ölproduktion gewonnenen Surplusprofite zugunsten der ölproduzierenden Staaten zu bestimmen. So sind die beiden Beiträge durchaus auf verschiedenen Ebenen der Analyse angesiedelt und könnten einander ergänzen. Dies geschieht auch in gewissem Rahmen. Während von Massarrat mit der Untersuchung der Wertbewegung in der Energiebranche die Grenzen für die Ölpreissteigerung und die Verteilungsverhältnisse des Surplusprofits mit Hilfe der Marxschen Grundrententheorie bestimmt werden, während für ihn also die Konkurrenz in der Energiebranche zwischen den verschiedenen Energieträgern (Steinkohle, Erdgas, Erdöl usw.) und die Verteilung von Surplusprofiten bestimmten Gesetzen unterworfen ist, behandelt Tahmassebi die Machtkonstellationen und das daraus resultierende Handeln der Ölförderländer, der Ölkonzerne und der Verbraucherländer. Daß gerade durch die Versuche aller Beteiligten, für sich möglichst viel herauszuholen, einen möglichst großen Anteil am Surplusprofit der Ölproduktion zu ergattern, die Gesetze, denen die Wirkungsweise der Konkurrenz unterworfen ist, durchgesetzt werden, bleibt den Beteiligten verborgen und wird von Tahmassebi nicht behandelt. In seiner Darstellung erscheinen die multinationalen Ölmonopole eher als Souveräne des Prozesses. Dennoch ist die Darstellung dieser Bewegungen sehr wichtig, ist es doch durchaus möglich, an einem derzeit politisch wichtigen Fall, Macht und Ohnmacht von Staaten gegenüber dem Kapital zu exemplifizieren.

In einem wichtigen Punkt widersprechen sich die Aufsätze. Dies liegt einmal an den unterschiedlichen analytischen Ebenen, auf denen sie ihre Argumentation entfalten, zum anderen aber auch an der Schwierigkeit, genaue Informationen über die konkrete Ölpreisbildung zu erhalten. Es ist klar, daß der Marktpreis des von den Ölförderländern in eigener Regie verkauften öls in den vergangenen Jahren, insbesondere aber seit dem 16.10.1973 erhöht worden ist. Umstritten allerdings ist folgende Frage: $O b$ die Erhöhung der Listenpreise - die der Berechnung der Abgaben der Ölkonzerne an die Förderländer zugrundegelegt werden - nur einem Vorschlag der Förderländer entspricht, der noch der verbindlichen Aushandlung mit 
den Ölkonzernen bedarf, also noch eine Machtauseinandersetzung nach sich zieht, deren Ausgang keineswegs klar ist (Tahmassebi) oder aber bereits realiter eingefordert wird, weil die Wirkungsweise des Wertgesetzes dies zuläßt (Massarrat). Zur Klärung der Kontroverse ist es nützlich, zwei Tabellen über die Zusammensetzung des Ölpreises anzufuihren (Neue Zürcher Zeitung vom 25. 1. 1974).

\begin{tabular}{|c|c|c|}
\hline & $\begin{array}{l}\text { Persischer Golf } \\
\text { "Arabian Light" } \\
34^{\circ} \text { API }\end{array}$ & $\begin{array}{l}\text { Libyen } \\
40^{\circ} \mathrm{API}\end{array}$ \\
\hline $\begin{array}{l}\text {,Posted Price" } \\
\text { Royalty } 12,5 \% \\
\text { Förderkosten }\end{array}$ & $\begin{array}{r}11.65 \\
1.46 \\
-.12\end{array}$ & $\begin{array}{c}15.768 \\
1.97 \\
-.40\end{array}$ \\
\hline Steuerbemessungsgrundlage & 10.07 & 13.40 \\
\hline $\begin{array}{l}\text { Steuersatz von } 55 \% \\
+ \text { zusätzlich Royalty }\end{array}$ & $\begin{array}{l}5.54 \\
1.46\end{array}$ & $\begin{array}{l}7.37 \\
1.97\end{array}$ \\
\hline $\begin{array}{l}\text { Direkte Steuer an Förderstaat } \\
\text { Förderkosten }\end{array}$ & $\begin{array}{r}7.00 \\
-.12\end{array}$ & $\begin{array}{r}9.34 \\
-.40\end{array}$ \\
\hline Selbstkosten der Gesellschaft & 7.12 & 9.74 \\
\hline
\end{tabular}

DIE ENTWICKLUNG DES ,POSTED PRICE“" ,Arabian Light“

(Saudiarabien)

(\$pro Faß)

Stichtag

1. 8.70

15. 2.71

20. 1.72

1. 1.73

1.10 .73

16.10 .73

1. 1.74
Posted Direkte Steuem

Price

1.80

2.18

2.48

2.59

3.01

5.12

11.65 an Förderland
Libyen $40^{\circ} \mathrm{API}$

Posted

Price

Direkte Steuern an Förderland

2.23

2.55

3.64

3.78

4.60

8.92

15.77
1.09

1.39

2.13

2.20

3.20

5.59

9.34 
Aus der ersten Tabelle geht die Unterschiedlichkeit der Preise je nach Fördergebiet hervor, wobei ein Teil der Unterschiedlichkeit auf Qualitätsunterschiede des o̊ls reduzierbar ist, ein anderer Teil aber auf eine unterschiedlich hohe Aneignung von Teilen des Surplusprofits durch die Förderländer. Bei einem einheitlichen Verkaufspreis des Öls und verschiedenen Selbstkosten der Konzerne sind die als Grundrente bezogenen Teile des Surplusprofits für die einzelnen Länder unterschiedlich. Daß dies von der politischen Macht der jeweiligen Staaten mit abhängt, ist durchaus einsichtig, wenn die Macht auch nicht allein entscheidend ist.

Die zweite Tabelle zeigt die Entwicklung des Listenpreises (posted price) seit 1970 und dokumentiert, daß, gleichgültig um welches Fördergebiet es sich handelt, dieser Freis permanent gestiegen ist und mit ihm auch die Abgaben an das Förderland. Die Tendenz, daß ein Teil des Surplusprofits nun den Förderländern in größerem Umfang zukommt, wird hier sehr deutlich.

So scheint sich die von Massarrat abgeleite te und begründete These zu bestätigen. Ein Gesichtspunkt allerdings bedarf der Erwähnung. Zunächst stellt sich der auf die Förderländer entfallende Teil des Surplusprofits in Geld, d. h. in der Regel in Form von Dollardevisen dar. Solange diese nicht gegen Waren aus den hochentwickelten kapitalistischen Verbraucherländern des öls eingetauscht sind, stellt der erhöhte Anteil am Surplusprofit nichts anderes dar als eine Vergrößerung des Kredits, den die armen ölförderländer den reichen Ölverbraucherländern gewähren. Die riesigen Dollarbeträge der arabischen Länder bei europäischen Banken sind nichts anderes als ein solcher riesiger Kredit, der nur insofern nachteilig für die hochentwickelten kapitalistischen Länder werden kann, als die Geldmassen von den arabischen Staaten selbst oder von den Banken, die mit ihnen ,arbeiten", spekulativ verschoben werden und Währungskrisen herbeiführen können.

Die Einlösung des Kredits kann nur in Waren erfolgen. So begründet sich u. a. auch die Forderung z. B. Saudi-Arabiens nach Produktionsmitteln zur Industrialisierung des Landes. Daher aber auch gleichzeitig die enormen Waffengeschäfte der arabischen Länder mit den hochentwickelten kapitalistischen Staaten wie erst jüngst das 800 Millionen Dollargeschäft: Öl gegen Waffen zwischen Saudi-Arabien und Frankreich. Die Möglichkeiten und Konsequenzen einer Industrialisierung und die Bedeutung der Aufrüstung solcher Regime wie im Iran oder in Saudi-Arabien als Ausdruck der neuen Verhältnisse im Energiesektor bedürfen noch einer eingehenden Analyse.

Auf ein Problem, das in beiden Aufsätzen nicht behandelt wird, aber für die politische Lage in den hochentwickelten kapitalistischen Ländern wichtig ist, soll hier noch kurz hingewiesen werden. Zwar wäre es falsch, die Energiekrise als ein bewußtes Manöver des internationalen Kapitals zu interpretieren, aber die Ausnutzung der zeitweisen Ölverknappung diente ganz eindeutig ,zum Vorwand einer psychologischen Notstandsdramarturgie ", durch die das „Krisenbewußtsein wach (gehalten wird), das wiederum die Betroffenen empfänglich machen soll für die wiederentdeckten Maßhalteappelle. Das zur ,Erdölkrise" stilisierte Embargo bietet ein eindrucksvolleres Szenarium für nationale Solidaritätsaufrufe oder gar für die Gewissenserforschung der Nation." So schreibt die Neue Zürcher Zeitung vom 17.1.1974. Wie richtig diese Einschätzung ist, wurde in den Tarifrunden von 
1973/74 mehr als deutlich gemacht; die Krise des Kapitalismus wurde flugs zur Ölkrise umdefiniert. Nicht mehr die Widersprüche des kapitalistischen Systems und der Klassengegensatz eklatieren in der Krise, sondern diese ist „,von außen durch die Araber aufgezwungen" worden und erfordert von allen das ihrige: von der Arbeiterklasse Lohnverzicht und von den Mineralölkonzernen enorme Profite, damit sie in der Zukunft ebenso enorme Investitionen finanziell bewältigen können (zum letzten vgl. die Argumentation in der Süddeutschen Zeitung vom 26./27. 1. 1974, S. 4).

Diese Reaktionsweise des bürgerlichen Staates im Verein mit den bürgerlichen Massenmedien ist durchaus nicht auf Westdeutschland beschränkt. Die Einführung der Dreitagewoche in England zur Niederwerfung der streikenden englischen Bergarbeiter wurde auch mit der ,ölkrise" begründet. Die Antwort der Bergarbeiter und der britischen Arbeiterklasse generell war bisher aber die einzig richtige Antwort darauf: der Notstandsdramaturgie der Heath-Regierung (z.B. durch militärische Besetzung des zivilen Flugplatzes London-Heathrow) die verschärfte Militanz der Kämpfe entgegenzusetzen und sich nicht mit ,Solidaritäts" ins Bockshorn kapitalistischer Krisenregulierung jagen zu lassen.

E. A.

\section{Die gr
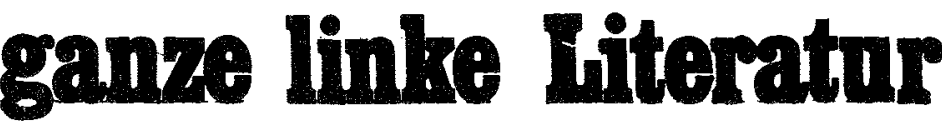

auch in Städten und Dörfern ohne sozialistische Buchhandlung, durch den Postversand des
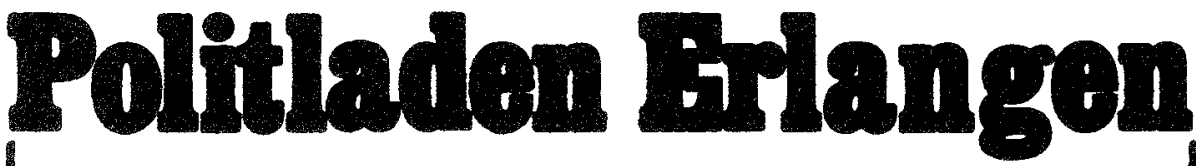

Klassiker des Marxismus, Theorie der Neuen Linken, Reprints und Archive, Geschichte der Arbeiterbewegung, Internationaler Klassenkampf; Bücher, Broschüren, Infos, Plakate und Schallplatten der sozialistischen Verlage des In- und Auslandes.

Versandlisten anfordern! Auslieferung aller Bestellungen portofrei auf Rechnung.

Postanschrift: POLITLADEN 852 ERLANGEN POSTFACH 2849 